\title{
Características epidemiológicas y clínicas de las cardiopatías congénitas en menores de 5 años del Hospital Almanzor Aguinaga Asenjo. Enero - Diciembre 2012.
}

\author{
Ana Karla Uribe Rivera ${ }^{1, a}$, Cristian Díaz-Vélez ${ }^{1,2, b}$, Carlos Cerrón-Rivera ${ }^{1,2, c}$
}

\section{RESUMEN}

Objetivo: Identificar las características epidemiológicas y clínicas de las cardiopatías congénitas en niños menores de 5 años en el Hospital “Almanzor Aguinaga Asenjo" durante enero - diciembre de 2012.

Material y Métodos: Estudio cuantitativo de diseño descriptivo transversal, población escogida niños menores de 5 años atendidos en Hospital Nacional "Almanzor Aguinaga Asenjo", diagnosticados con cardiopatías congénitas entre enero diciembre de 2012, los datos se obtuvieron del Sistema de Gestión Hospitalaria mediante el código CIE-10 (Q00-Q99) en la Oficina de Inteligencia Sanitaria del Hospital Nacional “Almanzor Aguinaga Asenjo" obteniéndose una lista con la cual se solicitó a la Oficina de Registros médicos las historias clínicas para la confección de la base de datos en Excel 2010.

Resultados: Se tuvo 109 pacientes, predominando sexo masculino (55\%) entre 6 meses y 2 años (40\%) procediendo de Lambayeque $(55 \%)$. Predominaron las cardiopatías congénitas acianóticas $(92 \%)$, siendo comunicación interventricular (CIV), comunicación interauricular (CIA) y persistencia del conducto arterioso (PCA) los más frecuentes, mientras que las cianóticas (8\%), el 56\% representóla Tetralogía de Fallot. Su tratamiento: 38\% farmacológico, 22\% observación y 40\% quirúrgico.

Conclusión: Las cardiopatías congénitas más prevalentes en el Hospital Nacional Almanzor Aguinaga Asenjo son similares a las reportadas en literatura nacional e internacional. Con mayor frecuencia en sexo masculino, procedentes de Lambayeque y según tipo, acianóticas, siendo comunicación interventricular en mayor proporción, y su tratamiento prevalece el conservador, siendo observación y farmacológico predominantemente. (Horiz Med 2015; 15(1): 49-56)

Palabras clave: Cardiopatías congénitas, características epidemiológicas, características clínicas. (Fuente: DeCS BIREME).

Epidemiological and clinical characteristics of congenital cardiopathies in children under 5 in Hospital Almanzor Aguinaga Asenjo. January - December 2012

\section{ABSTRACT}

Objective: Identify the epidemiological and clinical characteristics of congenital cardiopathies in children under 5 in “AlmanzorAguinagaAsenjo's" Hospital during January - December 2012.

Material and Methods: Quantitative descriptive cross sectional study design, the chosen were children under 5 years who were diagnosed with congenital cardiopathies in “AlmanzorAguinagaAsenjo's” Hospital between January to December 2012, data were obtained from the Hospital Management System using the ICD-10 (Q00-Q99) in the area of Health Intelligence of “ AlmanzorAguinagaAsenjo's " Hospital and obtained a list of which doctors office requested medical records for making database in Excel 2010.

Results: 109 patients, predominantly male (55\%) between 6 months and 2 years (40\%) from Lambayeque (55\%), predominated acyanotic congenital cardiopathies (92\%), ventricular septal defect (VSD ), atrial septal defect (ASD) and patent ductus arteriosus (PCA) the most common, whereas cyanotic congenital heart disease (8\% ), 56\% represented by Tetralogy of Fallot. Treatment, $38 \%$ with drug treatment, $22 \%$ observation and $40 \%$ surgical treatment.

Conclusion: The most prevalent congenital heart disease in the AlmanzorAguinagaAsenjo's Hospital are similar to those reported in national and international literature. More frequently in males, from Lambayeque and of the acyanotic type, being the interventricular communication most frequently, and treatment prevails conservative, observation and pharmacological depending on hemodynamic stability. (Horiz Med 2015; 15(1): 49-56)

Key words: congenital cardiopathies, epidemiological characteristics, clinical characteristics. (Source: MeSH NLM).

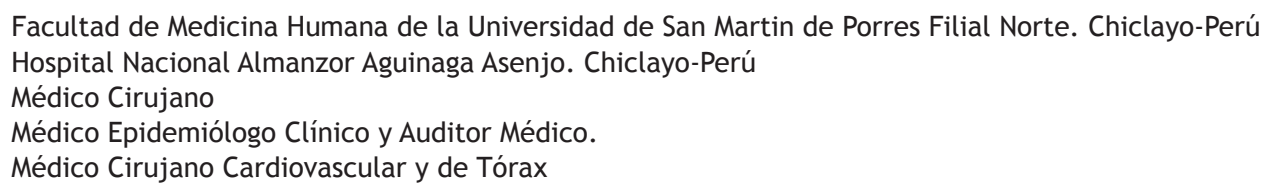




\section{INTRODUCCIÓN}

Las cardiopatías congénitas (CC) constituyen actualmente un importante problema de salud, algunas de éstas no repercuten gravemente en la vida diaria del paciente, mientras que otras son más serias, limitando la capacidad funcional y comprometiendo la calidad de vida, por lo que requieren, en la mayoría de casos, procedimiento quirúrgico en algún momento de la vida del paciente (1).

Existen numerosos estudios que establecen la incidencia de las cardiopatías congénitas, siendo el $0,8 \%$ la media (1), y a nivel global entre 0,7 y $0,9 \%(2,3)$, las cuales presentan variaciones dependientes del momento del diagnóstico, la población estudiada y los métodos diagnósticos utilizados.

En Estados Unidos $(4,6)$ se reporta según tipo de estudio y selección de datos un amplio rango de prevalencias, encontrándose desde 4 hasta 50 por 1,000 , estimándose actualmente entre 650,000 y $1,300,000$ adultos con malformaciones cardiacas congénitas y que este número asciende a un ritmo de $5 \%$ por año(2).

A nivel internacional, se llegó a determinar en distintos estudios y series de casos que los tipos de enfermedad tienen una distribución similar a la reportada por otros estudios, siendo de mayor prevalencia las cardiopatías de tipo acianóticas, donde las másfrecuentesfueron defectos del tabique interventricular e interauricular, persistencia del conducto arterioso, estenosis valvular pulmonar y coartación de aorta (2).

Del Mar Martín, Llanes, Castillo, González, Torres, Vega (2003) en su investigación: "Incidencia de las cardiopatías congénitas en el menor de un año. Villa Clara. 1998 -2002",diagnosticaron 466 niños con cardiopatía congénita de un total de 50.432 para una incidencia de 9.24/1.000 nacidos vivos cercano a lo estimado mundialmente.La persistencia del conducto arterioso y la comunicación interventricular tuvieron una elevada incidencia debido a su temprano diagnóstico (3).
Rodríguez, Ariza, Riaño, Moro, Suarez, Mosquera, García (2009) en su investigación titulada "Epidemiología de las cardiopatías congénitas en Asturias durante el período 1990-2004", la prevalencia total media fue de 75,2 por 10.000 nacidos, con una tendencia ascendente. Las más frecuentes fueron la comunicación interventricular (28,8 por cada 10.000 nacidos vivos), los defectos del septo auricular (10,3 por cada 10.000 nacidos vivos) y la persistencia del ductus arterioso (6,0 por cada 10.000 nacidos vivos). El 73,6\% de las cardiopatías congénitas se presentó de forma aislada, el 12,5\% asociada a otras anomalías congénitas y el $14 \%$ pertenecían a un síndrome o a una secuencia (9).

Rodríguez, Shoemaker (2007) en su investigación titulada:"Cardiopatías congénitas en niños menores de cinco años. Serie de casos, 20032006: Hospital Regional, Santander de Quilichao, Cauca, Colombia", se revisó 79 historias clínicas de niños con sospecha o diagnóstico de cardiopatía congénita. En 51 (64.6\%) de los pacientes se obtuvo un diagnóstico definitivo: cardiopatía congénita en $32(40.5 \%)$, y un soplo funcional en $19(24.1 \%)$. Cuatro niños (12.5\%) no volvieron para tratamiento; 24 (75\%) niños recibieron tratamiento médico inicial pero sólo $8(25 \%)$ fueron intervenidos quirúrgicamente (10).

En Perú, existe un estudio realizado en Lima, en el cual se da un aproximado de la realidad relacionada a las cardiopatías congénitas, Olórtegui, Adrianzén (2007), en su investigación: "Incidencia estimada de las cardiopatías congénitas en niños menores de 1 año en el Perú", entre los resultados se estimó que en el 2006 existieron 3925 cardiopatías, de las cuales dentro de los tipos de cardiopatía congénita, las acianóticas fueron las más frecuentes, representando aproximadamente el $83 \%$ de todas las cardiopatías congénitas, mientras que las cianóticas agrupan el $17 \%$.Las más frecuentes son comunicación interventricular (CIV) y comunicación interauricular (CIA), las que en conjunto representan aproximadamente el $50 \%$ de este grupo y el $35 \%$ de todas las cardiopatías. Por su parte, la cardiopatía congénita cianótica más frecuente es la tetralogía de Fallot, que representa el $70 \%$ de este grupo y alrededor del $10 \%$ de este grupo de afecciones. 
También, se estimó que representarían el 2,5\% del total de razón de años de vida potencialmente perdidos por cada mil habitantes (8).

En la cuidad de Chiclayo no se han encontrado estudios realizados sobre cardiopatías congénitas, el hospital de la red asistencial norte es el Hospital Nacional "Almanzor Aguinaga Asenjo", en el cual solo existen datos aislados sobre casos selectos, mas no estudios estadísticos completos sobre este tipo de patologías.

El objeto del estudio fue identificar las características epidemiológicas y clínicas de las cardiopatías congénitas en niños menores de 5 años en el Hospital “Almanzor Aguinaga Asenjo" durante enero - diciembre de 2012.

\section{MATERIAL Y MÉTODOS}

Estudio cuantitativo de diseño descriptivo transversal. La población escogida fueron niños menores de 5 años atendidos en el Hospital Nacional “Almanzor Aguinaga Asenjo", diagnosticados con cardiopatías congénitas entre enero - diciembre de 2012, cuyos datos se obtuvieron del Sistema de Gestión Hospitalaria mediante el código CIE-10 (Q20-Q28) en la Oficina de Inteligencia Sanitaria del Hospital Nacional "Almanzor Aguinaga Asenjo" obteniéndose una lista con la cual se solicitó a Oficina de Admisión y Registros médicos, las historias clínicas para la confección de la base de datos en Excel 2010.

\section{RESULTADOS}

Se obtuvo un total de 109 pacientes, entre las edades de 0 a 5 años en el transcurso de enero a diciembre del 2012, del total de los pacientes se determinó que el grupo etario más frecuente de diagnóstico fue entre los 6 meses hasta los 2 años (lactantes mayores) representando el $40 \%$ del total de pacientes, a su vez el sexo masculino representó el $55 \%$ de casos (Tabla 1 ).

Respecto a la procedencia, al ser un hospital referencial de la macrorregión, la mayor concentración de casos se obtuvo del mismo departamento de Lambayeque siendo el 55\% de los casos, siguiendo en frecuencia pacientes pertenecientes del departamento de Piura, con un $23 \%$ del total y en relación a la zona en que proceden, en un $80 \%$ pertenecieron al área urbana.

En la zona de Lambayeque, igualmente el predominio de tipo de cardiopatías fueron acianóticas, siendo el $50 \%$ de total, mientras que las cianóticas representaron el 6\% de la población de Lambayeque, siguiendo en secuencia Piura, igualmente predominando las de tipo acianóticas siendo el $22 \%$ de casos (Tabla 1 ).

Las cardiopatías más frecuentes fueron las de tipo acianóticas, corroborándose lo obtenido de estudios previos, siendo los defectos del tabique interventricular (CIV) e interauricular (CIA), persistencia del conducto arterioso (PCA), coartación de aorta los más frecuentes (2), así pues en nuestro estudio, represento el $92 \%$ del total, predominando en el sexo masculino siendo el $50 \%$ del total de casos (Tabla 2), de las cardiopatías acianóticas: comunicación interventricular, comunicación auricular y la persistencia del ductus arterioso son las tres más frecuentes conjuntamente siendo el $63 \%$ de casos, $13 \%$ de los casos de cardiopatías acianóticas fueron múltiples, siendo más frecuente la asociación comunicación interauricular (CIA) con comunicación interventricular (CIV) con un 7\%, mientras que las cardiopatías de tipo cianóticas se reportó $8 \%$ total, siendo la Tetralogía de Fallot el $56 \%$ de casos.

A su vez $75 \%$ de todos los casos se presentaron en forma aislada, predominando en el sexo masculino con $43 \%$ del total, a su vez $25 \%$ formaban parte de otra patología, en su mayoría a Trisomía 21 (Síndrome de Down) representando el 68\% de los casos de patologías asociadas.

En relación a su tratamiento en el $38 \%$ de casos se dio tratamiento farmacológico, mientras que $22 \%$ se mantuvo en observación y $40 \%$ necesito tratamiento quirúrgico, cuya relación con la inestabilidad hemodinámica repercutió en gran magnitud, donde el 33\% de casos de cardiopatías presentaban compromiso hemodinámico y se optó por la decisión quirúrgica. 
Respecto a la repercusión hemodinámica, la mayor parte de casos no tuvieron compromiso hemodinámico, abarcando el $67 \%$ del total de casos, a su vez el predominio de los casos donde se evidenció compromiso hemodinámico fue en el sexo femenino siendo $17 \%$ del total, la edad predominante donde se evidenciócompromiso hemodinámico fue entre los 29 días y 6 meses (lactantes menores) representando el 17\%, mientras que la edad predominante donde no se evidenció compromiso hemodinámico se encontró entre los 6 meses y 2 años de edad (lactantes mayores) respectivamente.
En relación a patologías asociadas y el compromiso hemodinámico, se determinó que a pesar que la mayor parte de pacientes presentaron casos aislados, $28 \%$ de estos presentaron repercusión hemodinámica, y dentro de los pacientes con patologías asociadas, el $4 \%$ de ellos fueron casos de pacientes con Trisomía 21 (Síndrome de Down) ya pesar que la mayor parte de pacientes no tuvo compromiso hemodinámico, los pacientes en los que hubo repercusión hemodinámica el $81 \%$ de estos pertenecieron a las de tipo acianóticas, mientras que el $19 \%$ de casos a las cianóticas.

Tabla 1. Características epidemiológicas de las cardiopatías congénitas en niños menores de 5 años del Hospital Almanzor Aguinaga Asenjo durante enero - diciembre 2012.

\section{Características Epidemiológicas}

Género

Masculino

Femenino

Edad de diagnóstico

$$
\begin{aligned}
& 0 \text { días - } 28 \text { días } \\
& 29 \text { días }-6 \text { meses } \\
& 6 \text { meses - } 2 \text { años } \\
& 2 \text { años - } 5 \text { años }
\end{aligned}
$$

Lugar de procedencia

Amazonas
Cajamarca
La Libertad
Lambayeque
Lima
Piura
San Martín
Tumbes

Zona de procedencia

Rural

Urbano

$$
n=109
$$$$
\mathrm{n}=\%
$$

$55 \%$

$45 \%$

$14 \%$

$39 \%$

$40 \%$

$7 \%$

$4 \%$

$8 \%$

$6 \%$

$55 \%$

$1 \%$

$23 \%$

$1 \%$

$2 \%$

$20 \%$

$80 \%$ 
Características epidemiológicas y clínicas de las cardiopatías congénitas en niños menores de 5 años del Hospital Almanzor Aguinaga Asenjo. durante Enero - Diciembre 2012.

Tabla 2. Características clínicas de las cardiopatías congénitas en niños menores de 5 años del Hospital Almanzor Aguinaga Asenjo durante enero - diciembre 2012

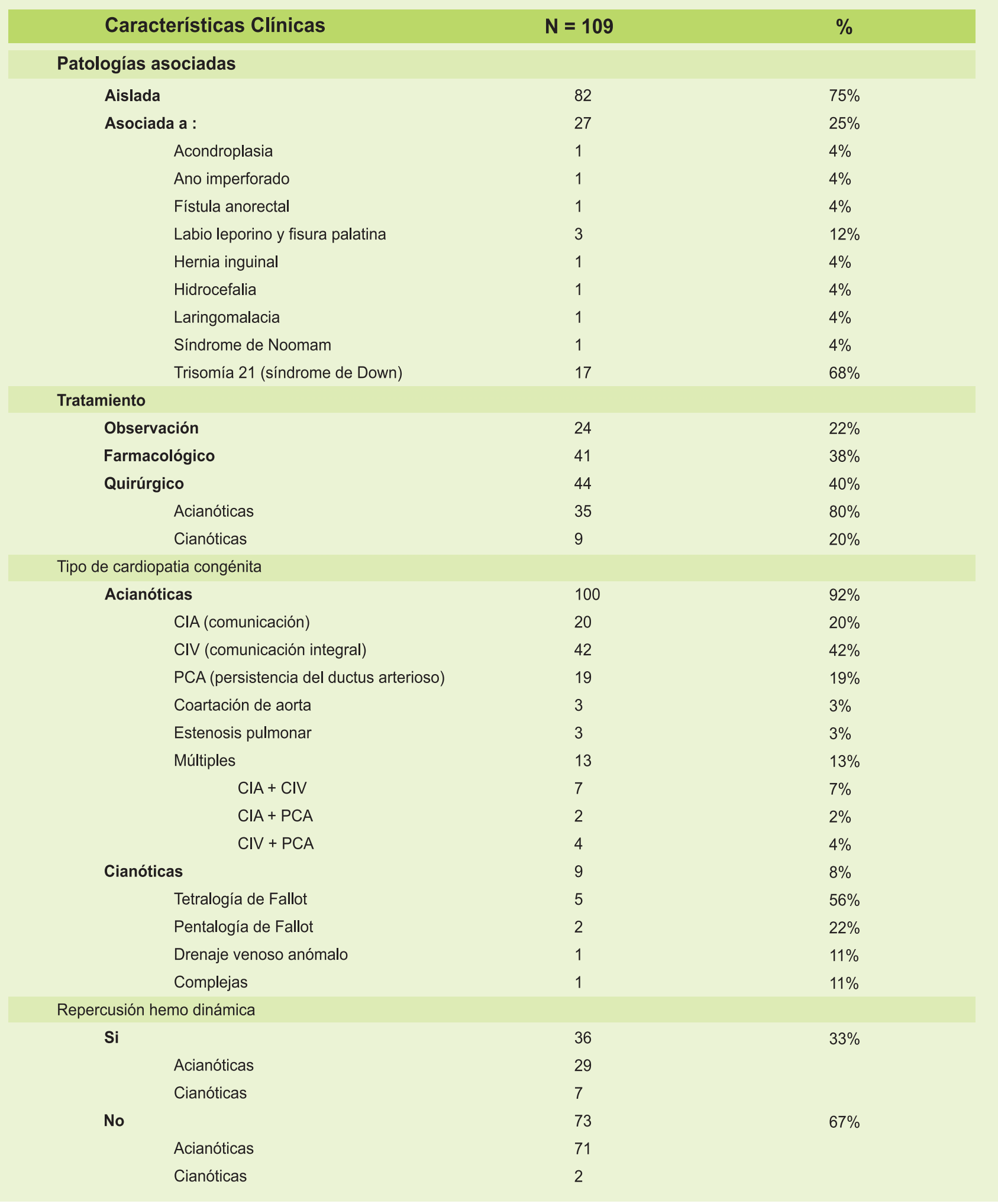




\section{DISCUSIÓN}

Según los resultados, la incidencia de cardiopatías congénitas en el Hospital Nacional Almanzor Aguinaga Asenjo está dentro del rango reportado a nivel mundial (11).

En relación al sexo (femenino/masculino) los resultados obtenidos fueron similares a la reportada en la literatura, donde la incidencia fue mayor en el sexo masculino, que varía entre $1: 1,3(12,13)$.

Según el grupo etario más frecuente de diagnóstico fue entre los 6 meses hasta los 2 años (lactantes mayores) representando el $40 \%$, lo que discrepa con diversos estudios internacionales dado a que, el promedio de edad al diagnóstico de cardiopatías congénitas es en menores de 6 meses, no obstante, con respecto a los países desarrollados, la edad de diagnóstico encontrada en nuestra población es un momento tardío pues no se cuenta con diagnóstico prenatal preciso y oportuno, así pues, en Francia $47,3 \%$ detodas las cardiopatías congénitas son diagnosticadas prenatalmente, mientras que en Baltimore (Estados Unidos), 90\% son diagnosticadas antes de los 7 meses de edad y en Latinoamérica solo el $24 \%$ son diagnosticadas después del egreso de las puérperas $(14,16)$.

A pesar ser un hospital referencial, la concentración de casos fue en su mayoría del departamento de Lambayeque, siendo el $55 \%$ del total, a su vez no hay diferencias significativas en la prevalencia de cardiopatías congénitas entre las áreas urbanas y rurales. El lugar y la zona de procedencia no fueron un factor asociado a la ocurrencia de cardiopatías congénitas en las diferentes áreas, pese que en nuestro estudio se obtuvo un resultado con predominio en la población urbana, y procedente de Lambayeque. Según datos internacionales esto no es un buen indicador ya que no evalúa el estatus socioeconómico en la población de casos de cardiopatías congénitas en específico (17).

La asociación entre cardiopatías congénitas y malformaciones extracardíacas en nuestro estudio es igual que la reportada a nivel mundial, a pesar que la proporción de pacientes es distinta a la evidenciada en diferentes estudios, su relación con otras patologías asociadas oscila entre $25 \%$, según el Baltimore-Washington Infant Study, y $27,5 \%$ según el estudio de Pradat $P$, que incluyó a 12932 niños con CC de 4,4 millones de nacimientos en tres grandes registros de California, Francia y Suecia $(18,19)$.

En relación al tipo de cardiopatía congénita más encontrada, nuestros resultados coinciden con lo reportado con Boneva RS et al (2001) donde se reporta el predominio de cardiopatías congénitas acianóticas y corroborando el predominio en el sexo masculino (20).

En el estudio realizado por Silva y Cols (21) la cardiopatía congénita más frecuente fue la Comunicación Interventricular (CIV) representado en $53,3 \%$, dato muy cercano al encontrado en este trabajo. Otro estudio realizado, señala un $61,2 \%$ (22) dato que también reafirma la alta incidencia de este tipo de cardiopatía. Magliola y colaboradores (23) reportaron que las patologías más frecuentes fueron la Comunicación Interventricular (CIV), Tetralogía de Fallot, y la Comunicación Interauricular (CIA) que comprenden casi el $50 \%$ de las cirugías.

Otros autores reportan Cardiopatías Congénitas en el $13,7 \%$ de los casos con síndromes genéticos y específicamente 5,8\% con Trisomía 21 (Síndrome de Down), alteración más frecuente (24), cuyo resultado que coincide con esta investigación y evidenciando en los casos de estos pacientes mayor predominio de compromiso hemodinámico, sin embargo el género no fue un factor predisponente, donde no se evidencio alguna diferencia significativa (21).

De acuerdo con lo obtenido se discrepa con datos internacionales sobre su tratamiento, en su mayoría es tipo conservador (observacional y farmacológico) con resultados positivos en el seguimiento y control continuos de pacientes a largo plazo (10), en nuestro estudio se evidenció que una proporción significativa de cardiopatías acianóticas necesitaron tratamiento quirúrgico, y a pesar de ser en menor proporción, su necesitad de un tratamiento mas complejo; esta diferencia se debe al diagnóstico mas tardío, 
que generalmente se da ante la evidencia de otro estado patológico (principalmente componente de tipo respiratorio) que condiciona a la exacerbación de la sintomatología y repercusión hemodinámica que abarca generalmente en edades menores de 1 año (25), que en relación a lo obtenido en este estudio se encuentra dentro del rango de edad en que predomina este componente.

Los resultados encontrados en comparación entre el compromiso hemodinámico y el tipo de cardiopatía mostraron resultados significativos, en donde se corrobora que a mayor complejidad el tipo de cardiopatía, el compromiso hemodinámico es mayor, asi pues, las cardiopatías de tipo cianóticas conllevan a un estado hemodinámico alterado, con alteración del flujo pulmonar, cardíaco y sistémico que repercuten en el estado general del paciente y subsecuente ante un pronóstico reservado en su evolución natural, por lo que en su gran mayoría necesitan de tratamiento quirúrgico para su resolución parcial o total (10).

\section{REFERENCIAS BIBLIOGRÁFICAS}

1. Aguilera P, Rodríguez A, Enríquez G, Vascopé X, Pizarro O, Almuna R. Cardiopatías congénitas con diagnóstico prenatal: seguimiento. RevChilObstetGinecol. [en Internet]. 2006; 74(5): 320-326 [citado 17 nov 2012]; Disponible en:ScientificElectronic Library Online: www.scielo.cl/pdf/rchog/v71n5/art05.pdf

2. Benavides A, Faerron J, Umaña L, Romero J.Epidemiología y registro de las cardiopatías congénitas en Costa Rica.RevPanam Salud Pública. [en Internet]. [citado 17 nov 2012]; Disponible en: ScientificElectronic Library Online: http://www.scielosp.org/pdf/ rpsp/v30n1/v30n1a05.pdf.

3. Del Mar M, Llanes M, Castillo A, González G, Torres D, VegaT. Incidencia de las cardiopatías congénitas en el menor de un año. Villa Clara. 1998 -2002.An.Rev Costarricense de Cardiología. [En Internet]. 2003. [citado 17 nov 2012]; Disponible en: ScientificElectronic Library Online: http://www.scielo.sa.cr/scielo. php?pid=S1409-41422003000100004\&script=sci_arttext

4. García D, Livengood L. Estudio retrospectivo realizado en el Departamento de Pediatría de la Unidad Nacional de Cirugía Cardiovascular de Guatemala -UNICAR-, 2005 al 2010 junio - agosto 2011. [Tesis en Internet]. Guatemala: Universidad San Carlos de Guatemala, Facultad de Medicina. 2011. [accesado 15 de noviembre 2012]. Disponible en internet: http:// biblioteca.usac.edu.gt/ tesis/05/05_8802.pdf.

5. Hernández R, Fernández-Collado C, Baptista, P. Metodología de la investigación. 4ta ed. México: Mc Graw-Hill. 2008.

6. Heusser, F. Problemas frecuentes en cardiología pediátrica. Revchilpediatr. [en internet] 2009: 1,4-6,8 [citado 17 nov
Las limitaciones de este estudio fueron los datos incompletos de algunas historias clínicas, la disponibilidad de las mismas para su recolección de datos y la dificultad de contactar algunos pacientes.

En los casos de incorrecta codificación de CIE-10, la existencia como segundo diagnóstico de cardiopatía congénita, de haber existido esto, no se tomaron en cuenta estos casos.

Es de suma importancia que en las unidades de atención materno infantil se integre un equipo multidisciplinario formado por el obstetra, neonatólogo, cardiólogo pediatra, cirujano pediatra, epidemiólogo y el psicopedagogo para la detección y atención médica y quirúrgica del recién nacido con cardiopatías congénitas y su familia, considerando los avances actuales en diagnóstico y cirugía fetal, en donde las opciones de resolución cada vez son más amplias y seguras; así mismo con el objeto de disminuir la tasa de mortalidad.
2012]; Disponible en: Escuela de Medicina: http:// escuela.med.puc.cl/ paginas/ publicaciones/ manualped/ cardiocong. htm

7. Kuehl K, Loffredo A, Ferencz C. Failure to diagnose congenital heart disease in infancy. Pediatrics [en Internet]. 1999.103(4): 743-7 [citado 17 nov 2012]; Disponibleen:Official journal of the American Academy of Pediatrics:http://pediatrics.aappublications.org/ content/103/4/743.abstract

8. Olortegui A, Adrianzen M. Incidencia estimada de las cardiopatías congénitas en niños menores de 1 año en el Perú. An. Fac. med. [en Internet]. 2007 abr./jun., vol.68, no.2, p.113-124. [citado 15 Feb 2012]; Disponible en: ScientificElectronic Library Online: http:// www.scielo.org.pe/pdf/afm/v68n2/a03v68n2.pdf.

9. Rodríguez C, Ariza F, Riaño I. Epidemiologia de la enfermedad congénita cardiaca en Asturias (España) durante el periodo 1990 - 2004. AnPediatr (Barc). [en Internet]. 2009 jun; 71(6): 5002-9. [citado 17 nov 2012]; Disponible en Elservier: http://www.elsevier. es/es/revistas/anales-pediatria-37/epidemiologia-las-cardiopatiascongenitas-asturias-durante-periodo-13145293-originales2009?bd=1.

10. Rodríguez V, Shoemaker R. Cardiopatías congénitas en niños menores de cinco años. Serie de casos., 2003-2006: Hospital Regional, Santander de Quilichao, Cauca, Colombia.[Tesis en Internet]. Cauca: Universidad del Cauca, Facultad de Salud; 2007. [accesado 18 de nov 2012]. Disponible en internet: http: / / www.facultadsalud.unicauca.edu.co/fcs/2007/junio/ cardiopat\%C3\%ADas\%20cong\%C3\%A9nitas.pdf 
11. Hoffman JIE. Incidence of congenital heart disease: I. Postnatal incidence. PedatrCardiol 1995; 16: 103-13

12. Samanek M. Boy:Girl ratio in children with different forms of cardiac malformation: a population-based study. Pediatr Cardiol.1994;15(2):53-7.

13. Laursen HB. Some epidemiological aspects of congenital heart disease in Denmark. ActaPaediatr Scand. 1980;69(5):619-24.

14. Memberg A, OtterstadJE, Froland G, Hals J, Sorland SJ. Early clinical screening of neonates for congenital heart defects: the cases we miss. Cardiol Young. 1999;9(2):169-74.

15. Kuehl K, Loffredo C, Ferencz C. Failure to diagnose congenital heart disease in infancy. Pediatrics. 1999;103(4):743-8.

16. Correa-Villaseñor $M$, McCarter R, Downing J, Ferencz C. The Baltimore-Washington Infant Study Group. White-black differences in cardiovascular malformations in infancy and socioeconomic factors. Am J Epidemiol. 1991;134(4):393-403.

17. Ministerio de Planificación y Política Económica (MIDEPLAN), Costa Rica. Índice de desarrollo social, 2007. San José, Costa Rica: MIDEPLAN, 2007.30. Vrijheid M, Dolk H, Stone

18. Pradat P, Francannet C, Harris JA, Robert E. The Epidemiology of Cardiovascular Defects, Part 1: a study based on data from three large registries of congenital malformations. PediatrCardiol. 2003;24(3):195-221.

19. Ferencz C, Correa-Villaseñor A, Loffredo CA, Wilson PD. Genetics and enviromental risk factors of major cardiovascular malformations:
The Baltimore-Washington Infant Study: 1981-1989. Perspectives in Pediatric Cardiology, Vol. 5. Armonk, NY: Futura Publishing;1997.

20. Boneva RS, Botto LD, Moore CA, Yang Q, Correa A, Erickson JD. Mortality associated with congenital heart defects in the United States: trends and racial disparities, 1979-1997. Circulation 2001; 103: 2376-81.

21. Blanco E.; Almeida S; Russinyoll G; Rodríguez G; Olivera E; Medina E. Actualización sobre cardiogénesis y epidemiología de las cardiopatías congénitas. Revista médica electrón; 31(3), mayojunio.2009.

22. Silva VM, Lopes MVO, Araujo TL. Evaluación de los percentiles de crecimiento en niños con cardiopatías congénitas. Rev. Latino-am Enfermagem 2007 marzo-abril; 15(2)

23. Magliola R, Althabe M, Charroqui A, Moreno G, Balestrini M, Landry L et al. Cardiopatía congénita: actualización de resultados quirúrgicos en un hospital pediátrico 1994-2001. Arch. argent. pediatr. 2004 mar. / abr, 102(2).

24. González TR. Características generales de las cardiopatías congénitas en niños del municipio Cerro. Año 2006. Tesis Maestría. Facultad de Ciencias Médicas Dr. "Salvador Allende". Ciudad Habana. 2006. 89 p.

25. Hoffman JIE: Incidence of congenial heart desease. II: Prenatal incedence. PediatrCardiol 1995:16: 155-165.

\section{Fuentes de financiamiento}

Autofinanciado por la autora.

\section{Conflictos de interés}

\section{La autora declara no tener ningún conflicto de interés}

\section{Correspondencia:}

Ana Karla Uribe Rivera

Dirección: Calle El Sendero 238 - Urb. 3 de

Octubre Chiclayo, Perú.

Teléfono: (51) 982091443

Correo electrónico: kauri-1.6@hotmail.com 